\title{
Topologically-Protected Qubits from a Possible Non-Abelian Fractional Quantum Hall State
}

\author{
Sankar Das Sarma ${ }^{1}$, Michael Freedman ${ }^{2}$, Chetan Nayak $^{2,3}$ \\ ${ }^{1}$ Department of Physics, University of Maryland, College Park, MD 20742 \\ ${ }^{2}$ Microsoft Research, One Microsoft Way, Redmond, WA 98052 \\ ${ }^{3}$ Department of Physics and Astronomy, University of California, Los Angeles, CA 90095-1547
}

(Dated: September 10, 2018)

\begin{abstract}
The Pfaffian state is an attractive candidate for the observed quantized Hall plateau at Landau level filling fraction $\nu=5 / 2$. This is particularly intriguing because this state has unusual topological properties, including quasiparticle excitations with non-Abelian braiding statistics. In order to determine the nature of the $\nu=5 / 2$ state, one must measure the quasiparticle braiding statistics. Here, we propose an experiment which can simultaneously determine the braiding statistics of quasiparticle excitations and, if they prove to be non-Abelian, produce a topologically-protected qubit on which a logical NOT operation is performed by quasiparticle braiding. Using the measured excitation gap at $\nu=5 / 2$, we estimate the error rate to be $10^{-30}$ or lower.
\end{abstract}

Introduction The computational power of a quantummechanical Hilbert space is potentially far greater than that of any classical device [1, 2]. However, it is difficult to harness it because much of the quantum information contained in a system is encoded in phase relations which one might expect to be easily destroyed by its interactions with the outside world ('decoherence' or 'error'). Therefore, error-correction is particularly important for quantum computation. Fortunately, it is possible to represent information redundantly so that errors can be diagnosed and corrected [3, 4].

An interesting analogy with topology suggests itself: local geometry is a redundant way of encoding topology. Slightly denting or stretching a surface such as a torus does not change its genus, and small punctures can be easily repaired to keep the topology unchanged. Only large changes in the local geometry change the topology of the surface. Remarkably, there are states of matter for which this is more than just an analogy. A system with many microscopic degrees of freedom can have ground states whose degeneracy is determined by the topology of the system. The excitations of such a system have exotic braiding statistics, which is a topological effective interaction between them [5]. Such a system is said to be in a topological phase [6, 7]. The unusual characteristics of quasiparticles in such states can lead to remarkable physical properties, such as a fractional quantized Hall conductance [8]. Such states also have intrinsic fault-tolerance [9]. Since the ground states are sensitive only to the topology of the system, interactions with the environment, which are presumably local, cannot cause transitions between ground states unless the environment supplies enough energy to create excitations which can migrate across the system and affect its topology. When the temperature is low compared to the energy gap of the system, such events will be exponentially rare.

A different problem now arises: if the quantum information is so well-protected from the outside world, then how can we - presumably part of the outside world - manipulate it to perform a computation? The answer is that we must manipulate the topology of the system. In this regard, an important distinction must be made between different types of topological phases. In the case of those states which are Abelian, we can only alter the phase of the state by braiding quasiparti- cles. In the non-Abelian case, however, there will be a set of $g>1$ degenerate states, $\psi_{a}, a=1,2, \ldots, g$ of particles at $x_{1}, x_{2}, \ldots, x_{n}$. Exchanging particles 1 and 2 might do more than just change the phase of the wavefunction. It might rotate it into a different one in the space spanned by the $\psi_{a}$ s:

$$
\psi_{a} \rightarrow M_{a b}^{12} \psi_{b}
$$

On the other hand, exchanging particles 2 and 3 leads to $\psi_{a} \rightarrow M_{a b}^{23} \psi_{b}$. If $M_{a b}^{12}$ and $M_{a b}^{23}$ do not commute (for at least some pairs of particles), then the particles obey non-Abelian braiding statistics. In the case of a large class of states, the repeated application of braiding transformations $M_{a b}^{i j}$ allows one to approximate any desired unitary transformation to arbitrary accuracy and, in this sense, they are universal quantum computers [10]. Unfortunately, no non-Abelian topological states have been unambiguously identified so far. Some proposals have been put forward for how such states might arise in highly frustrated magnets [11, 12], where such states might be stabilized by very large energy gaps on the order of magnetic exchange couplings, but the best prospects in the short run are in quantum Hall systems, where Abelian topological phases are already known to exist. The best candidate is the quantized Hall plateau with $\sigma_{x y}=\frac{5}{2} \frac{e^{2}}{h}$. The $5 / 2$ fractional quantum Hall state (as well as its particle-hole symmetric analog, the $7 / 2$ state) is now routinely observed [13] in high-quality (i.e. low-disorder) samples. In addition, extensive numerical work [14] using finite-size diagonalization and wavefunction overlap calculations indicates that the $5 / 2$ state belongs to the non-Abelian topological phase characterized by a Pfaffian quantum Hall wavefunction [15, 16]. The set of transformations generated by braiding quasiparticle excitations in the Pfaffian state is not computationally universal (i.e. is not dense in the unitary group), but other non-Abelian states in the same family are. Thus, it is important to (a) determine if the $\nu=5 / 2$ state is, indeed, in the Pfaffian universality class and, if so, to (b) use it to store and manipulate quantum information. In this paper, we propose an experimental device which can address both of these. Features of our device are inspired by anti-dot experiments measuring the charge of quasiparticles [17] in Abelian fractional quantum Hall states such as $\nu=1 / 3$ and proposals for measuring their statistics 
[18]. Our measurement procedure relies upon quantum interference as in the electronic Mach-Zehnder interferometer in which Aharonov-Bohm oscillations were observed in a twodimensional electron gas [19].

In order to establish which topological phase the $\nu=5 / 2$ plateau is in, one must directly measure quasiparticle braiding statistics. Remarkably, this has never been done even in the case of the usual $\nu=1 / 3$ quantum Hall plateau (although in this case, unlike in the $\nu=5 / 2$ case, computational solutions of small systems leave little doubt about which topological phase the plateau is in). Part of the problem is that it is difficult to disentangle the phase associated with braiding from the phase which charged particles accumulate in a magnetic field [18]. Ironically, it may actually be easier to measure the effect of non-Abelian braiding statistics because it is not just a phase and is therefore qualitatively different from the effect of the magnetic field.

Pfaffian Facts To make this latter point clear, let us summarize some important properties of quasiparticles in the Pfaffian state. The Pfaffian state may be viewed as a quantum Hall state of $p$-wave paired fermions. The quasiparticles in this phase have charge- $e / 4$ (not $e / 2$, as one might naively assume from the Landau-level filling fraction $\nu=2+\frac{1}{2}$; this emphasizes the importance of an experiment such as [17] to measure the quasiparticle charge at $\nu=5 / 2$ ). When there are $2 n$ quasiparticles at fixed positions in the system, there is a $2^{n-1}$-dimensional degenerate space of states. Exchanging and braiding quasiparticles is related to the action of the $2 n$-dimensional Clifford algebra on this space [20], as has recently been confirmed by direct numerical evaluation of the Berry matrices [21]. In particular, two charge-e/4 quasiparticles can 'fuse' to form a charge- $e / 2$ quasiparticle either with or without a neutral fermion in its core. One may view the charge-e/2 quasiparticle as the quantum Hall incarnation of a superconducting vortex with a fermionic zero mode in its core [22, 23, 24, 25]. We will regard the presence or absence of a neutral fermion in this core state if the two charge- $e / 4$ quasiparticles were fused as our qubit. So long as the two quasiparticles are kept far apart, the neutral fermion is not localized anywhere and, therefore, the qubit is unmeasurable by any local probe or environment. However, we can measure the qubit by encircling it with a charge-e/4 quasiparticle. The presence of the neutral fermion causes the state to acquire an extra factor of -1 during this process. The qubit can also be manipulated by taking another charge- $e / 4$ quasiparticle between the two charge-e/4 quasiparticles comprising the qubit, i.e. around one but not the other. Such a process transforms a state without a neutral fermion into a state with one and vice versa. Thus, it flips the qubit (and also multiplies by $i$ ). By performing an experiment which measures this qubit, flips it, and then re-measures it, we can demonstrate that the $\nu=5 / 2$ state is in a non-Abelian topological phase. (A few additional similar experiments would be necessary to fully nail down that it is in the Pfaffian phase rather than another non-Abelian phase.) Such an experiment can only work if the environment does not flip the qubit before we have a chance to measure it, so the success of this experiment would demonstrate the stability of a topological qubit in a non-Abelian quantum Hall state. By varying the time between measurements, one could determine the decoherence time of the qubit in order to quantitatively compare it with other approaches to quantum computation.

The claimed quasiparticle braiding properties can be seen from the form of the four-quasihole wavefunctions given in [20]. The ground state wavefunction takes the form [15, 16]

$$
\Psi_{\text {g.s. }}\left(z_{j}\right)=\prod_{j<k}\left(z_{j}-z_{k}\right)^{2} \prod_{j} e^{-\left|z_{j}\right|^{2} / 4} \cdot \operatorname{Pf}\left(\frac{1}{z_{j}-z_{k}}\right) .
$$

where the Pfaffian is the square root of the determinant of an antisymmetric matrix. If we write

$$
\begin{gathered}
\Psi_{(13)(24)}\left(z_{j}\right)=\prod_{j<k}\left(z_{j}-z_{k}\right)^{2} \prod_{j} e^{-\left|z_{j}\right|^{2} / 4} \times \\
\operatorname{Pf}\left(\frac{\left(z_{j}-\eta_{1}\right)\left(z_{j}-\eta_{3}\right)\left(z_{k}-\eta_{2}\right)\left(z_{k}-\eta_{4}\right)+(j \leftrightarrow k)}{z_{j}-z_{k}}\right)
\end{gathered}
$$

and similarly for $\Psi_{(14)(23)}$, then the four-quasihole wavefunctions can be written in a basis in which their braiding is completely explicit:

$$
\Psi^{(0,1)}\left(z_{j}\right)=\frac{\left(\eta_{13} \eta_{24}\right)^{\frac{1}{4}}}{(1 \pm \sqrt{x})^{1 / 2}}\left(\Psi_{(13)(24)} \pm \sqrt{x} \Psi_{(14)(23)}\right)
$$

where $\eta_{13}=\eta_{1}-\eta_{3}$, etc. and $x=\eta_{14} \eta_{23} / \eta_{13} \eta_{24}$. Let us suppose that the quasiholes at $\eta_{1}$ and $\eta_{2}$ form our qubit. The quasiholes at $\eta_{3}$ and $\eta_{4}$ will be used to measure and manipulate them. From (4), we see that taking $\eta_{3}$ around $\eta_{1}$ and $\eta_{2}$ results in a factor $i$ in the state $\Psi^{(0)}$ but $-i$ in the state $\Psi^{(1)}$. Taking $\eta_{3}$ around either $\eta_{1}$ or $\eta_{2}$ (but not both) transforms $\Psi^{(0)}$ into $i \Psi^{(1)}$ and vice versa.

It is also possible [26] to verify the logic associated to braiding operations using a few formal properties of the Jones polynomial at $q=\exp (\pi i / 4)$. Taking one quasiparticle around the qubit pair ('linking') results in an extra -1 if the qubit is in state $|1\rangle$ (a factor $d=-q-q^{-1}$ also arises regardless of whether or not the quasiparticle encircles the qubit). The Jones polynomial (operator) at $q=\exp (\pi i / 4)$ vanishes for the links in figures 1 $1, \mathrm{~b}$ by calculation, 1, by parity, and is non-vanishing only for $1 \mathrm{~d}$ (which applies to all processes with topologically-equivalent link diagrams, e.g. interchanging inputs/outputs so, for example, 1 $1 \mathrm{~d}$ corresponds to four different processes). In case $1 \mathrm{~d}$, the qubit is flipped by the elementary braid operation.

Experimental Configuration The basic setup which we propose is a quantum Hall bar with two individually-gated anti-dots in its interior, labeled 1 and 2 in figure 2 There are front gates which enable tunneling between $\mathrm{A}$ and $\mathrm{B}$ at the edges. It is useful to have a third anti-dot at the point $\mathrm{C}$ midway between $A$ and $B$ in order to precisely control the charge which tunnels between $\mathrm{A}$ and $\mathrm{B}$, but we have not depicted it 
(a)

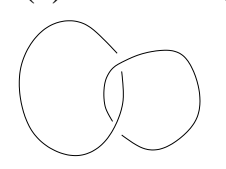

(b)

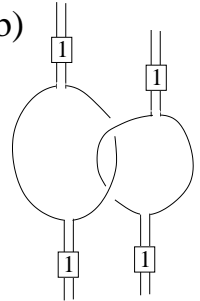

(c)

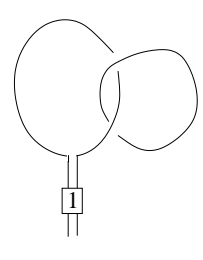

(d)

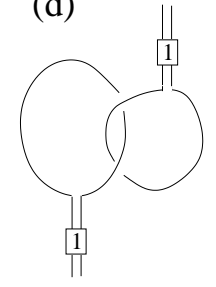

FIG. 1: By evaluating the Jones polynomial at $q=\exp (\pi i / 4)$ for these links, we can obtain the desired matrix elements for braiding operations manipulating the qubit. The boxed 1 is a projector on the pair of quasiparticles which puts them in the state $|1\rangle$.

to avoid clutter. Two more front gates enable tunneling at $\mathrm{M}$ and $\mathrm{N}$ and at $\mathrm{P}$ and $\mathrm{Q}$. There are three basic procedures which we would like to execute: (1) initialize the qubit and measure its initial state, (2) flip the qubit, and (3) measure it again.

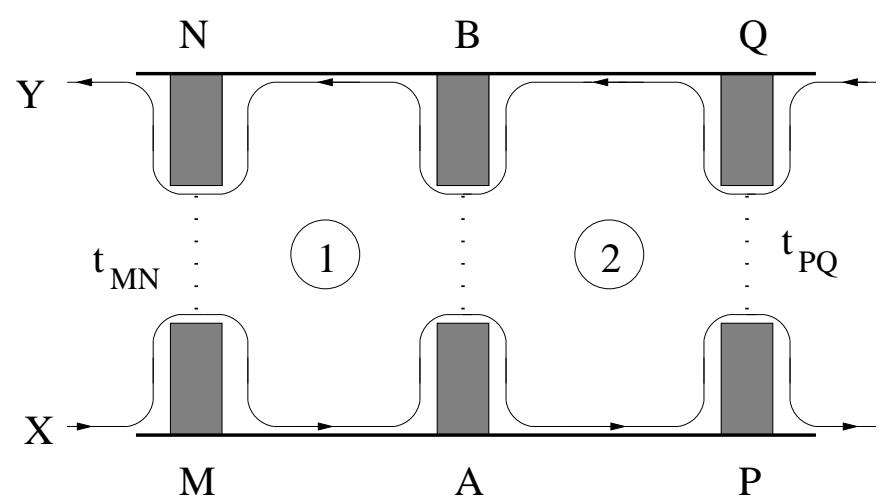

FIG. 2: A schematic depiction of a Hall bar with front gates which enable tunneling between the two edges at M, N and P, Q, thereby allowing a measurement of the qubit formed by the correlation between anti-dots 1 and 2. Front gates (shaded regions) also allow tunneling at $\mathrm{A}, \mathrm{B}$ which flips the qubit.

In order to initialize the qubit, we first put charge $e / 2$ on one of the antidots, say 1 . Since the fermionic zero mode is now localized on this antidot, the environment will 'measure' it, and it will either be occupied or unoccupied (not a superposition of the two). We can determine which state it is in by applying voltage to the front gates at $\mathrm{M}$ and $\mathrm{N}$ and at $\mathrm{P}$ and $\mathrm{Q}$ so that tunneling can occur there with amplitudes $t_{M N}$ and $t_{P Q}$. The longitudinal conductivity, $\sigma_{x x}$ is determined by the probability for current entering the bottom edge at $\mathrm{X}$ in figure 2 to exit along the top edge at $\mathrm{Y}$. This is given, to lowest order in $t_{M N}$ and $t_{P Q}$, by the interference between two processes: one in which a quasiparticle tunnels from $\mathrm{M}$ to $\mathrm{N}$; and another in which the quasiparticle instead continues along the bottom edge to $\mathrm{P}$, tunnels to $\mathrm{Q}$, and then moves along the top edge to $\mathrm{N}$. (We subsume into $t_{P Q}$ the phase associated with the extra distance travelled in the second process.) The relative phase of these processes depends on the state of the qubit. If a neutral fermion is not present, which we will denote by $|0\rangle$, then $\sigma_{x x} \propto\left|t_{M N}+i t_{P Q}\right|^{2}$. If it is present, however, which we denote by $|1\rangle$, then $\sigma_{x x} \propto\left|t_{M N}-i t_{P Q}\right|^{2}$. We take the visibility of Aharonov-Bohm oscillations in a device with similar limitations [19] (e.g. the possibility of the tunneling quasiparticles becoming dephased by their interaction with localized two-level systems) as an indication that our proposed read-out procedure will work.

Without loss of generality, let us suppose that the initial state of the qubit is $|0\rangle$. Now, let us apply voltage to anti-dots 1 and 2 so that charge $e / 4$ is transferred from 1 to 2 . There is now one charge- $e / 4$ quasihole on each anti-dot. The state of the qubit is unaffected by this process. In order to flip this qubit, we now apply voltage to the front gates at $\mathrm{A}$ and $\mathrm{B}$ so that one charge $e / 4$ quasiparticle tunnels between the edges. In order to ensure that only a single quasiparticle tunnels, it is useful to tune the voltage of the anti-dot at $\mathrm{C}$ and the backgate at $\mathrm{A}$ so that a single quasiparticle tunnels from the edge to the anti-dot at C. (If the anti-dot is small, its charging energy will be too high to allow more than one quasiparticle to tunnel at once.) We can then lower the voltage of the backgate at A so that no further tunneling can occur there and apply voltage to the backgate at $\mathrm{B}$ so that the quasiparticle can tunnel from $\mathrm{C}$ to $\mathrm{B}$. By this two-step process, we can tunnel a single quasiparticle from A to B. If the $\nu=5 / 2$ plateau is in the phase of the Pfaffian state, this will transform $|0\rangle$ to $|1\rangle$. This is our logical NOT operation. The gate which creates the anti-dot at $\mathrm{C}$ must be turned off at the beginning and end of the bit flip process so that there are no quasiparticles there either before or after which could become entangled with our qubit.

We can now measure our qubit again by tuning the front gates so that tunneling again occurs between $\mathrm{M}$ and $\mathrm{N}$ and between $\mathrm{P}$ and $\mathrm{Q}$ with amplitudes $t_{M N}$ and $t_{P Q}$. If, as expected, the qubit is now in the state $|1\rangle$ we will find $\sigma_{x x} \propto$ $\left|t_{M N}-i t_{P Q}\right|^{2}$. On the other hand, if the $\nu=5 / 2$ state were Abelian, $\sigma_{x x}$ would not be affected by the motion of a quasiparticle from A to B.

In order to execute these steps, it is important that we know that we have one (modulo 4) charge-e/4 quasihole on each anti-dot. This can be ensured by measuring the tunneling conductance $G_{t}^{\text {ad }}$ from one edge to the other through each antidot [17]. As we sweep the magnetic field, there will be a series of peaks in $G_{t}^{\text {ad }}$ corresponding to the passage through the Fermi level of quasihole states of the antidot. The spacing $\Delta B$ between states is determined by the condition that an additional state passes through the Fermi level when one additional half-flux-quantum, $\Phi_{0} / 2$ is enclosed in the dot. Thus, the number of quasiholes is given simply by $\lfloor B / \Delta B\rfloor$. Alternatively, with a back gate, we could directly measure capacitatively the charge on each anti-dot [17]. If the back gate voltage is $V_{B G}$ (relative to the zero quasihole case when the gate defining the anti-dot is turned off), then the charge on the anti-dot is $q=\epsilon V_{B G} A / d$, where $A=\Phi_{0} / 2 \Delta B$ is the area of the dot, $\epsilon$ the dielectric constant, and $d$ the distance between the back gate and the 2DEG.

Estimate of Error Rate Bit flip and phase flip errors, respectively, occur when an uncontrolled charge-e/4 quasiparticle performs one of the two basic processes above: encir- 
cling one of the anti-dots (or passing from one edge to the other between them) or encircling both of them. The rate for these processes is related to the longitudinal resistivity (which vanishes within experimental accuracy) because it is limited by the density and mobility of excited quasiparticles. Even without considering the suppression factor associated with the latter (which depends on the ratio of the diffusion or hopping length, $a$, to the system size, $L$ ), we already have a strong upper bound on the error rate following from the thermallyactivated form of the former (in $k_{B}=1$ units):

$$
\frac{\Gamma}{\Delta} \sim \frac{T}{\Delta} e^{-\Delta / T}<10^{-30}
$$

Here, we have used the best current measured value [27] for the quasiparticle gap $\Delta=500 \mathrm{mK}$ of the $5 / 2$ state and the lowest achieved measurement temperature $T=5 \mathrm{mK}$. For arbitrary braid-based computation, in a more elaborate device, it is sufficient if we further have $e^{\Delta / T}>\nu \Delta L^{2}$, where $\nu$ is the density-of-states. The effect of residual pinned quasiparticles can be diagnosed and accounted for in software. These error rates are substantially lower than the estimated error rate for any other physical implementations of quantum computation in any proposed architectures. Compared to other scalable solid state architectures, such as localized electron spin qubits [28] in $\mathrm{Si}$ or GaAs nanostructures, where the estimated error rate is around $10^{-4}$ even in the best possible circumstances, the errors associated with $\nu=5 / 2$ quantum Hall anyons is essentially negligible. This miniscule error rate arises from the intrinsic robustness of the topological phase which is fundamentally immune to all local environmental perturbations.

The ideal error rate for the $5 / 2$ state may actually be substantially lower than even this very low currently achievable value of $10^{-30}$. There is strong theoretical evidence [29] that the ideal excitation gap $(\sim 2 \mathrm{~K})$ for the $5 / 2$ quantum Hall state is much larger than the currently achieved gap value of $500 \mathrm{mK}$. Using an ideal gap of $2 \mathrm{~K}$, we get an astronomically low error rate of $10^{-100}$. This expected higher value of $\Delta$ ( $\sim 2 \mathrm{~K})$ is consistent with the experimental development of the activation gap measurement [13] of the $5 / 2$ state. The early measurements on fairly modest quality samples (i.e. relatively highly disordered) gave $\Delta \sim 100 \mathrm{mK}$ whereas recent measurements in extremely high-quality (i.e. low disorder) samples give $\Delta \sim 300-500 \mathrm{mK}[13]$. This implies that the $5 / 2$ excitation gap is susceptible to strong suppression by disorder as has recently been theoretically argued [29]. Since improvement in sample quality has already led to a factor of 5 enhancement in $\Delta$ (from $100 \mathrm{mK}$ to $500 \mathrm{mK}$ ), it is not unreasonable to expect further improvements.

There are, in principle, other sources of error, but we expect them to be of minor significance. For example, if two quasiparticles come close to each other, then their mutual interaction leads to an error (e.g. through the exchange of a virtual particle). Such a virtual exchange is, however, a quantum tunneling process which should be exponentially suppressed. Therefore keeping the quasiparticles reasonably far from each other should essentially eliminate this error.
We note that, although we have discussed only the $5 / 2$ Pfaffian quantized Hall state throughout this paper, our considerations and arguments apply equally well to the experimentally often-observed $7 / 2$ quantized Hall state which, being the 'hole' analog of the $5 / 2$ state by virtue of the particlehole symmetry, should have equivalent topological and nonAbelian properties. We believe the $5 / 2$ state to be a better experimental candidate for topological quantum computation because the measured excitation gap in the $5 / 2$ state tends to be much higher than that in the $7 / 2$ state. We should also mention that recently [13] the $12 / 5$ fractional quantum Hall state has been observed experimentally in the highest mobility sample at the lowest possible temperatures. This state, thought to be a non-Abelian state related to parafermions [30], is particularly exciting from the perspective of topological quantum computation because its braid group representation is dense in the unitary group [10] making this state an ideal candidate for topological quantum computation. The measured gap value in the $12 / 5$ state is currently rather small $(\sim 70 \mathrm{mK})$, making any experimental effort along the line of our discussion in this paper premature at this stage. However, we expect that this is also strongly affected by disorder and that the eventual ideal gap at $12 / 5$ will be much larger.

Acknowledgements We would like to thank J. Eisenstein, A. Kitaev, C. Marcus, and W. Pan for discussions. We have been supported by the ARO under Grant No. W911NF-04-10236. C. N. has also been supported by the NSF under Grant No. DMR-0411800.

[1] P. W. Shor, Proc. 35th Ann. Symp. on Found. of Comp. Sci., IEEE Computer Soc. Press., Los Alamitos, CA, 1994, 124-134.

[2] M. A. Nielsen and I. L. Chuang, Quantum Computation and Quantum Information (Cambridge University Press, Cambridge, 2000).

[3] P. W. Shor, Phys. Rev. A 52, R2493 (1995).

[4] D. Gottesman, Phys. Rev. A 57, 127 (1998).

[5] J. Leinaas and J. Myrheim, Nuovo Cimento 37B, 1 (1977); G. Goldin et al., J. Math. Phys. 22, 1664 (1981); F. Wilczek, Phys. Rev. Lett. 48, 1144 (1982). See also the discussion in Fractional Statistics and Anyon Superconductivity, edited by F. Wilczek (World Scientific, Singapore 1990).

[6] X. G. Wen, Int. J. Mod. Phys. B 4, 239 (1990).

[7] X. G. Wen, Q. Niu, Phys. Rev. B 41, 9377 (1990).

[8] Perspectives in Quantum Hall Effects: Novel Quantum Liquids in Low-Dimensional Semiconductor Structures, ed. by S. Das Sarma and A. Pinczuk (Wiley, New York, 1997).

[9] A. Kitaev, Ann. Phys. 303, 2 (2003).

[10] M. H. Freedman et al., Commun. Math. Phys. 227, 605 (2002).

[11] M. Freedman et al., cond-mat/0309120

[12] M. A. Levin and X.-G. Wen, cond-mat/0404617

[13] J.S. Xia et al., Phys. Rev. Lett. 93, 176809 (2004); J.P. Eisenstein et al., PRL 88, 076801(2002); W. Pan et al., Phys. Rev. Lett. 83, 3530 (1999); J.P. Eisenstein et al., Surf. Sci. 229, 31(1990); R.L. Willett et al., Phys. Rev. Lett. 59, 1776(1987).

[14] R. H. Morf, Phys. Rev. Lett. 80, 1505 (1998). E. H. Rezayi, F. D. M. Haldane, Phys. Rev. Lett. 84, 4685 (2000).

[15] G. Moore and N. Read, Nucl. Phys. B 360, 362 (1991).

[16] M. Greiter et al., Nucl. Phys. B 374, 567 (1992). 
[17] V. J. Goldman and B. Su, Science 267, 1010 (1995).

[18] C. de C. Chamon et al., Phys. Rev. B 55, 2331 (1997).

[19] Y. Ji et al., Nature 422, 415 (2003).

[20] C. Nayak and F. Wilczek, Nucl. Phys. B 479, 529 (1996).

[21] Y. Tserkovnyak and S. H. Simon, Phys. Rev. Lett. 90, 016802 (2003)

[22] N. Read and E. Rezayi, Phys. Rev. B 54, 16864 (1996).

[23] N. Read and D. Green, Phys. Rev. B 61, 10267 (2000).

[24] D. A. Ivanov, Phys. Rev. Lett. 86, 268 (2001).
[25] A. Stern et al., Phys. Rev. B 70, 205338 (2004).

[26] E. Fradkin et al., Nucl. Phys. B 516, 704 (1998).

[27] W. Pan, private communication.

[28] S. Das Sarma et al., cond-mat/0411755

[29] R. Morf and N. d'Ambrumenil, Phys. Rev. B 68, 113309 (2003); R.H. Morf et al., Phys. Rev. B 66, 075408 (2002).

[30] N. Read and E. Rezayi, Phys. Rev. B 59, 8084 (1999). 